\title{
Função do fixador externo circular de Ilizarov no tratamento de fraturas tibiais com síndrome compartimental iminente/incompleta*
}

\section{The Role of the Ilizarov Ring External Fixator in the Management of Tibia Fractures with Impending/Incomplete Compartment Syndrome}

\author{
Kaushik Bhowmick ${ }^{1}$ Chandrasekaran Gunasekaran ${ }^{1}{ }^{10}$ Jacob J. Kandoth ${ }^{1}$ Bo Boopalan Ramasamy ${ }^{10}$ \\ Thilak S. Jepegnanam ${ }^{10}$ \\ 1 Departmento de Ortopedia, Christian Medical College and Hospital, \\ Vellore, Tamilnadu, Índia \\ Rev Bras Ortop 2021;56(5):579-587. \\ Endereço para correspondência Chandrasekaran Gunasekeran, \\ MS in Orthopaedics, Department of Orthopedics - Unit 3, Paul Brand \\ Building, Christian Medical College, Vellore, Tamilnadu, India \\ (e-mail: chandras.guna@gmail.com).
}

\section{Resumo}

Palavras-chave

- técnica de llizarov

- fraturas da tíbia

- síndromes compartimentais
Objetivo $\mathrm{O}$ tratamento das fraturas da tíbia complicadas por síndrome compartimental afeta o tratamento e o resultado funcional dos pacientes devido às complicações associadas à fasciotomia. O objetivo deste estudo é diferenciar a síndrome compartimental iminente/incompleta $(\mathrm{SCl})$ da síndrome compartimental aguda (SCA) estabelecida nas fraturas tibiais, para avaliar o resultado da fixação do aparelho de llizarov nos pacientes fraturados e com $\mathrm{SCl}$, que não foram submetidos à fasciotomia.

Métodos Após o estabelecimento dos critérios de inclusão e exclusão, 19 pacientes foram incluídos no estudo de janeiro de 2007 a dezembro de 2017. Todos eram do sexo masculino, com média de idade de 42,3 $\pm 11,38$ anos. Todos esses pacientes foram tratados com a fixação do aparelho de llizarov, de acordo com o protocolo médico e cirúrgico estabelecido neste estudo.

Resultados $\mathrm{O}$ acompanhamento médio dos nossos 19 pacientes foi de $47 \pm 41,5$ meses. O tempo médio de aplicação do fixador circular foi de 3,7 \pm 1 ,7 dias. No total, 3 $(16,7 \%)$ desses pacientes não apresentaram consolidação. Não houve complicações nas partes moles ou neurovasculares no pós-operatório imediato. A consolidação ocorreu finalmente em todos os pacientes, sem prejuízo da mobilidade e sem sequela de síndrome compartimental.

Trabalho desenvolvido no Departamento de Ortopedia, Christian Medical College and Hospital, Vellore, Índia

recebido

02 de Dezembro de 2020

aceito

19 de Fevereiro de 2021
DOI https://doi.org/

$10.1055 / \mathrm{s}-0041-1732332$ ISSN 0102-3616. (c) 2021. Sociedade Brasileira de Ortopedia e Traumatologia. All rights reserved.

This is an open access article published by Thieme under the terms of the Creative Commons Attribution-NonDerivative-NonCommercial-License, permitting copying and reproduction so long as the original work is given appropriate credit. Contents may not be used for commercial purposes, or adapted, remixed, transformed or built upon. (https://creativecommons.org/ licenses/by-nc-nd/4.0/)

Thieme Revinter Publicações Ltda., Rua do Matoso 170, Rio de Janeiro, RJ, CEP 20270-135, Brazil 


\begin{abstract}
Objective The management of tibia fractures complicated by compartment syndrome affects the treatment and functional outcome of patients due to the complications associated with fasciotomy. The purpose of the present study is to differentiate impending/incomplete compartment syndrome (ICS) from established acute compartment syndrome (ACS) in tibial fractures, and to assess the outcome of the fixation of the llizarov apparatus in patients with these fractures presenting with ICS, who were not submitted to fasciotomy.

Methods After the establishment of the inclusion and exclusion criteria, 19 patients were included in the study from January 2007 to December 2017. All patients were male, with an average age of $42.3 \pm 11.38$ years. All of these patients were managed with llizarov ring fixation as per the medical and surgical protocol established in the present study.

Results The average follow-up obtained for our 18 patients was of $47 \pm 41.5$ months, with one patient being lost to follow-up. The average time for ring application was of $3.7 \pm 1.7$ days. In total, $3(16.7 \%)$ of these patients had nonunion. There were no soft-tissue or neurovascular complications in the immediate postoperative period. All of the patients eventually united, and were independently mobile without any sequelae of compartment syndrome.

\section{Keywords}

- Ilizarov technique

- tibial fractures

- compartment syndromes

Conclusion The llizarov ring external fixator can be used in the management of tibial fractures with ICS, avoiding fasciotomy with its various complications of infection and nonunion, resulting in fewer surgeries and faster rehabilitation. Surgeons should carefully differentiate ACS and ICS in these patients, as the clinical and functional results vary significantly. Unnecessary fasciotomies should be avoided.
\end{abstract}

\section{Introdução}

O tratamento das fraturas tibiais complicadas por síndrome compartimental pode ter resultados clínicos e funcionais desfavoráveis. ${ }^{1}$ Até mesmo a intervenção oportuna da fasciotomia pode provocar infecção do sítio cirúrgico e retardo na consolidação da fratura, principalmente em pacientes tratados com fixação interna. ${ }^{2}$ Estu$\operatorname{dos}^{1,3}$ mostram que as medidas de pressão estão incorretas em até 30\% dos pacientes diagnosticados com síndrome compartimental. Nesses pacientes, fazer uma decisão incontestável pela realização de fasciotomia é difícil, em virtude das implicações médico-legais, até mesmo quando há tendência indicativa da realização de fasciotomia.

Em determinado subgrupo de pacientes nem todas as características clínicas da síndrome compartimental são manifestadas com a realização do exame clínico, e eles são diagnosticados como portadores de síndrome compartimental iminente/incompleta (SCI). Os critérios para diagnosticar a SCI são: aumento de leve a moderado na circunferência da perna; edema duro à palpação; surgimento de múltiplas bolhas cheias de líquido; dor leve ao alongamento dos tendões dos músculos que passam pelo compartimento envolvido; ausência de dor em repouso; e nenhum déficit vascular, sensorial ou motor. ${ }^{4,5}$

Este estudo teve como objetivo avaliar o resultado do uso do fixador externo circular de Ilizarov em pacientes com fraturas tibiais que apresentam SCI e não foram submetidos a uma fasciotomia.

\section{Pacientes e Métodos}

Este estudo retrospectivo foi aprovado pelo Conselho de Revisão Institucional. De janeiro de 2007 a dezembro de 2017, foram identificados 19 pacientes em nossos registros hospitalares, que foram incluídos neste estudo após a obtenção do consentimento. Todos os pacientes eram do sexo masculino $(n=19)$, com média de idade de 42,30 $\pm 11,38$ anos, envolvidos em traumas de alta energia. Dos 19 pacientes, 15 apresentavam fraturas fechadas, e 4 , fraturas expostas. Havia um paciente com hipertensão, e dois eram tabagistas.

Os critérios de inclusão foram:

1. Fraturas tibiais agudas fechadas/expostas, com ou sem extensão intra-articular, com sinais de $\mathrm{SCI}$, conforme descrito por Sood et al. ${ }^{4}$ 
2. Atividade mental normal. ${ }^{5}$

Os excluídos foram pacientes com:

1. Sensório alterado/Escala de Coma de Glasgow (ECG) $<15$;

2. Lesão vascular; e

3. Síndrome compartimental aguda (SCA) estabelecida.

\section{Características dos Pacientes e das Fraturas}

Os padrões de fratura e lesões das partes moles foram classificados de acordo com a classificação da Arbeitsgemeinschaft für Osteosynthesefragen (Grupo de Trabalho para Questões de Fusão Óssea)/Orthopedic Trauma Association (Associação de Trauma Ortopédico) (AO/OTA). ${ }^{6}$ Dos 19 pacientes, 16 (84,2\%) apresentaram fraturas intra-articulares cominutivas, 2 (10,5\%) pacientes apresentaram fraturas extra-articulares da tíbia proximal, e 1 (5,2\%) paciente apresentou fratura segmentar da diáfise tibial. As radiografias pré-operatórias foram realizadas em dois planos em todos os pacientes. A tomografia computadorizada foi realizada em 2 pacientes (Pacientes 2 e 16), pois eles apresentavam nas radiografias significativa depressão articular e cominuição. Os detalhes demográficos e das fraturas dos pacientes são mostrados na - Tabela 1.

\section{Tratamento Clínico}

Nosso protocolo de tratamento clínico foi seguido por todos os pacientes, e consistie na administração intravenosa (IV) de $100 \mathrm{ml}$ de manitol a $20 \%$ por um período de 24 a 72 horas, aplicação de pasta de sulfato de magnésio, elevação do membro, e aplicação da placa de gesso de Paris solta acima do joelho, a fim de reduzir o edema do membro. ${ }^{7,8} \mathrm{O}$ monitoramento musculoesquelético e neurovascular contínuo foi realizado com a finalidade de identificar os sinais de progressão para a síndrome compartimental. A presença de bolhas hemorrágicas indicou a gravidade da lesão das partes moles e o desluvamento interno. Nenhum dos pacientes $(\mathrm{n}=19)$ apresentou qualquer lesão neurovascular pré-operatória, e todos foram submetidos à fixação do aparelhol de Ilizarov em uma média de 3,7 $\pm 1,7$ dias após a lesão (-Fig. 1).

\section{Técnica Cirúrgica}

Nos pacientes com fraturas intra-articulares, a congruência da superfície articular da tíbia proximal foi alcançada com a aplicação de tração e incisões mínimas, a fim de elevar os fragmentos deprimidos com o auxílio de um osteótomo ou perfurador ósseo. A redução foi então mantida temporariamente com fios de Kirschner e finalmente com parafusos interfragmentários. O comprimento e o alinhamento do membro foram mantidos por meio de tração contínua e visualização por meio de um intensificador de imagens (II). O anel proximal do aparelho de Ilizarov foi fixado à tíbia proximal no nível da cabeça da fíbula, com seis pontos de aderência ao osso (três fios); então, dois anéis foram fixados nos terços médio e inferior da tíbia, com pelo menos quatro pontos de aderência ao osso. A estabilidade do aparelho foi ainda melhorada com o acréscimo de pinos de Schanz aos anéis, às vezes adicionando um anel de fêmur distal, que foi removido em seis semanas em todo os casos.
Em pacientes com fraturas tibiais metadiafisárias, o membro foi estabilizado com um ou dois anéis, dependendo da distância da fratura da linha da articulação do joelho de cada lado, mantendo o comprimento e o alinhamento do membro. Os anéis mais próximos à fratura no aparelho de Ilizarov foram fixados a pelo menos 1 polegada da linha da fratura, tanto proximal quanto distalmente.

No período pós-operatório, todos os pacientes foram mobilizados com carga parcial, que foi aumentada para carga total após 6 semanas. 0 aparelho de Ilizarov foi removido quando os pacientes conseguiam andar de forma confortável e sem dor, com padrão de marcha normal e as radiografias mostrando a consolidação. Após a remoção, os pacientes foram imobilizados com gesso de apoio para tendão patelar por um período de seis semanas.

\section{Análise dos Resultados}

A consolidação óssea foi definida radiologicamente como a consolidação de ao menos três córtices nas projeções anteroposterior (AP) e de perfil durante o período de acompanhamento. ${ }^{9}$ Os desfechos clínicos foram medidos pela pontuação da Knee Society (Knee Society Score, KSS, em inglês) ${ }^{10}$ e pela pontuação de desfecho de lesão no joelho e osteoartrite (Knee Injury and Osteoarthritis Outcome Score, KOOS, em inglês), ${ }^{11}$ ao passo que o desfecho radiológico foi medido pela pontuação funcional de Rasmussen. ${ }^{12}$ Foram observadas também todas as complicações da não consolidação, da lesão das partes moles, e da infecção no trajeto dos pinos. ${ }^{13}$

\section{Resultados}

Dos 19 pacientes, 1 (Paciente 1) faleceu de causas naturais após a consolidação óssea, e outro (Paciente 6) perdeu o acompanhamento. Neste estudo, apresentamos os resultados dos 18 pacientes disponíveis (incluindo o Paciente 1). A duração mediana do acompanhamento foi de $47 \pm 41,5$ meses. Todos os pacientes foram submetidos a fixação do aparelho de Ilizarov em uma média de $3,7 \pm 1,7$ dias após a lesão. Todos os 18 pacientes apresentaram consolidação, sem prejuízo da mobilidade, incluindo o paciente que havia falecido de causas naturais. (-Figs. 2, 3, 4, 5). Nenhum dos pacientes apresentou complicações neurovasculares pósoperatórias.

\section{Resultados Clínicos e Funcionais}

Os resultados clínicos e funcionais foram medidos pelas pontuações KSS e KOOS em todos os 17 pacientes (excluindo o paciente que faleceu). Os componentes clínicos e funcionais da pontuação KSS foram excelentes em 13 pacientes, bons em 3, e regulares em 1 (paciente 17). A pontuação KOOS também foi aceitável em todos, exceto 1 paciente (paciente 17), conforme mostrado na - Tabela 1.

\section{Resultados Radiológicos}

O resultado radiológico foi avaliado pela pontuação funcional de Rasmussen, que consiste de três componentes: depressão articular, alargamento condilar, e angulação, com classificação 


\begin{tabular}{|c|c|c|c|c|c|c|c|c|c|c|c|c|}
\hline 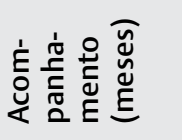 & 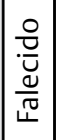 & $\stackrel{0}{m}$ & $\stackrel{6}{\circ}$ & $\simeq$ & $\stackrel{\infty}{\longrightarrow}$ & 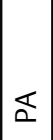 & $\approx$ & 우 & $\stackrel{\sim}{\sim}$ & $\stackrel{0}{m}$ & $\simeq$ & ஓి \\
\hline 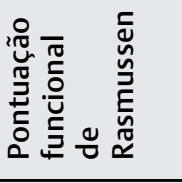 & $\Sigma$ & 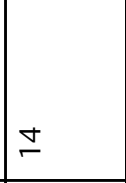 & 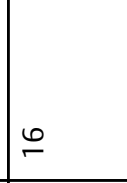 & 6 & 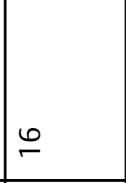 & $\Sigma$ & $\Sigma$ & $\stackrel{\infty}{\sim}$ & 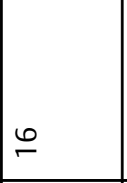 & 6 & 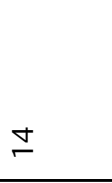 & 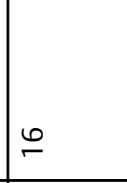 \\
\hline 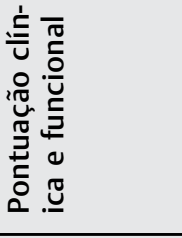 & $\S$ & 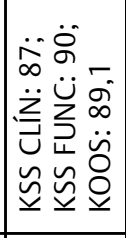 & 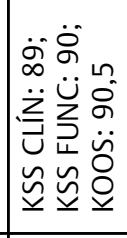 & 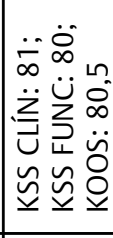 & 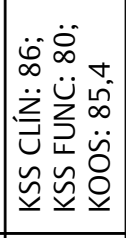 & $\Sigma$ & 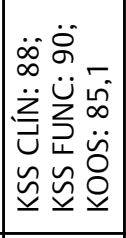 & 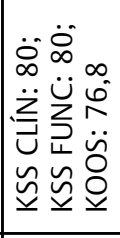 & 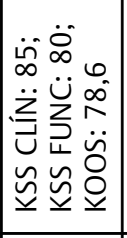 & 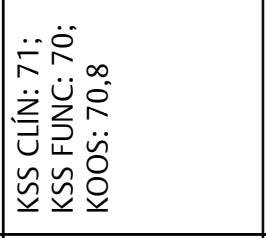 & 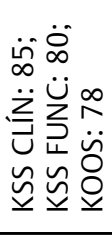 & 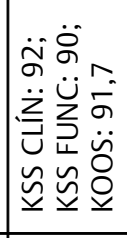 \\
\hline 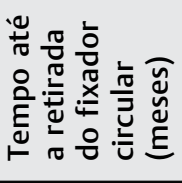 & $\wedge$ & 6 & 6 & $\wedge$ & $\nabla$ & & $\infty$ & $\infty$ & $m$ & $\infty$ & $\nabla$ & $m$ \\
\hline 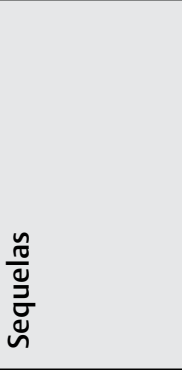 & 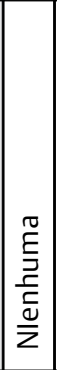 & 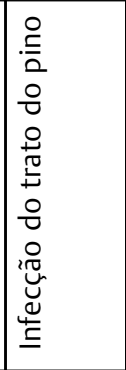 & 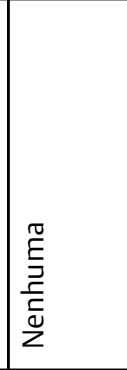 & 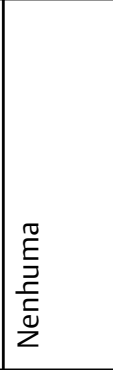 & 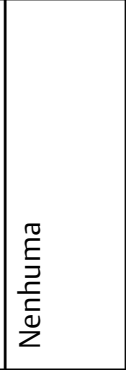 & 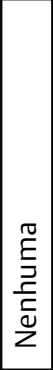 & 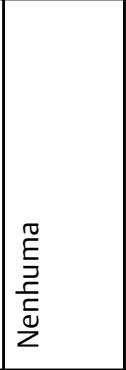 & 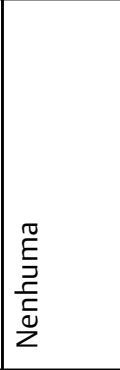 & 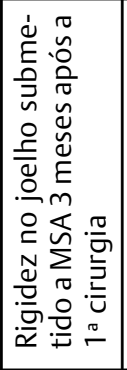 & 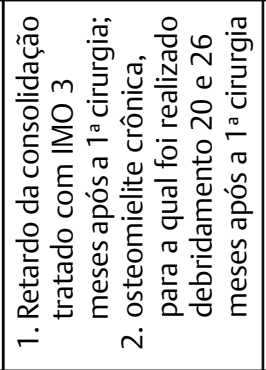 & $\begin{array}{l}\stackrel{0}{E} \\
\underline{\underline{D}} \\
\frac{1}{c} \\
\frac{D}{2} \\
z\end{array}$ & 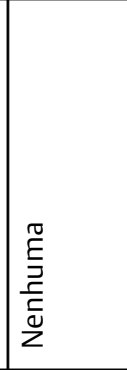 \\
\hline 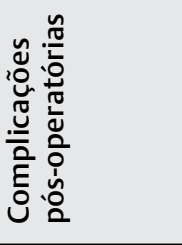 & 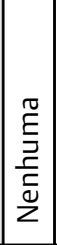 & $\overrightarrow{\bar{z}}$ & 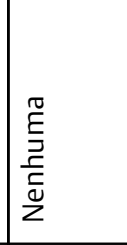 & 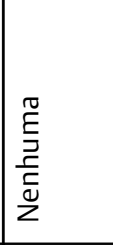 & 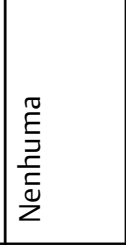 & 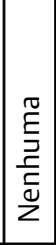 & 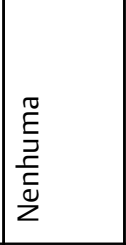 & 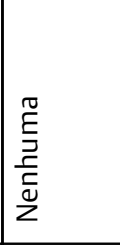 & 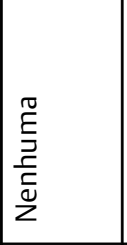 & 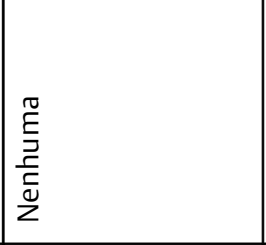 & $\begin{array}{l}\stackrel{0}{E} \\
\underline{\Xi} \\
\frac{1}{c} \\
\text { DU } \\
z\end{array}$ & 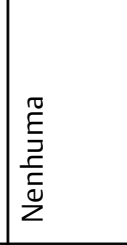 \\
\hline 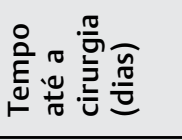 & $\nabla$ & 6 & $\sim$ & $m$ & 0 & $\nabla$ & $m$ & $\nabla$ & in & $\nabla$ & 0 & in \\
\hline$\stackrel{n}{\mathbb{D}_{\tilde{N}}} z$ & 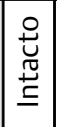 & 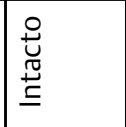 & 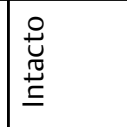 & 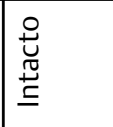 & 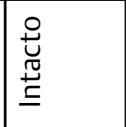 & 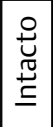 & $\begin{array}{l}\text { 导 } \\
\stackrel{\text { I }}{E}\end{array}$ & 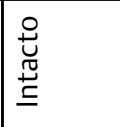 & 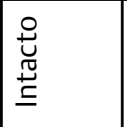 & 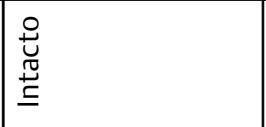 & 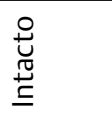 & 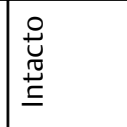 \\
\hline 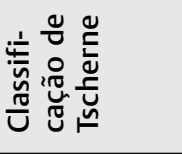 & $\sim$ & $\sim$ & $\sim$ & $\sim$ & $\sim$ & $\sim$ & $\sim$ & $\sim$ & $\sim$ & - & $\sim$ & $\sim$ \\
\hline 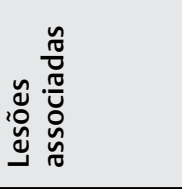 & 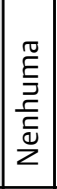 & 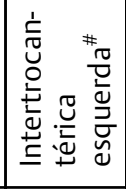 & 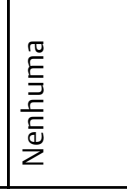 & 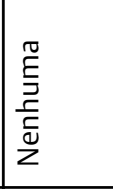 & 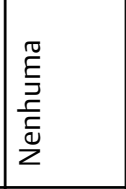 & 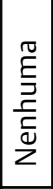 & 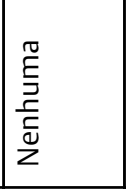 & 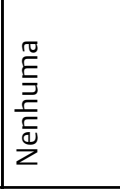 & 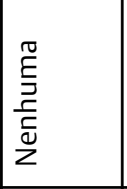 & 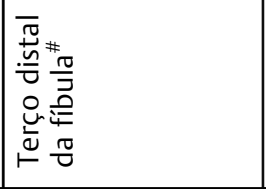 & 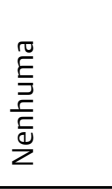 & 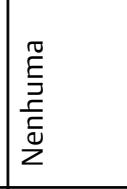 \\
\hline 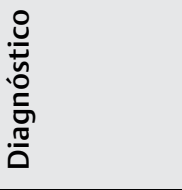 & 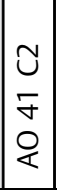 & $\begin{array}{l}0 \\
\frac{8}{8} \\
0\end{array}$ & $\begin{array}{l}\bar{z} \\
\bar{z} \\
\dot{\alpha}\end{array}$ & $\mid \begin{array}{l}\frac{1}{2} \\
\frac{2}{2} \\
0\end{array}$ & 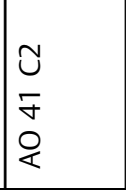 & 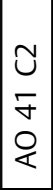 & 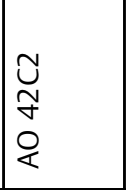 & 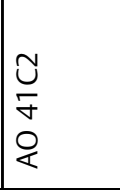 & 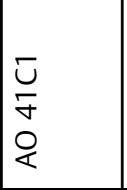 & 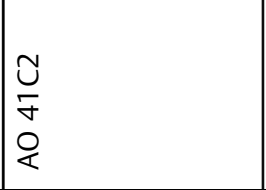 & $\begin{array}{l}\text { Ũ } \\
\frac{\gamma}{\sigma} \\
0 \\
<\end{array}$ & $\begin{array}{l}\bar{z} \\
\bar{z} \\
\dot{\alpha} \\
\end{array}$ \\
\hline 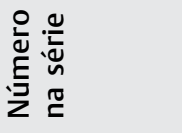 & - & $\sim$ & $m$ & 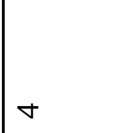 & เn & 6 & $r$ & $\infty$ & $a$ & $\stackrel{\circ}{\circ}$ & $\mp$ & $\simeq$ \\
\hline
\end{tabular}




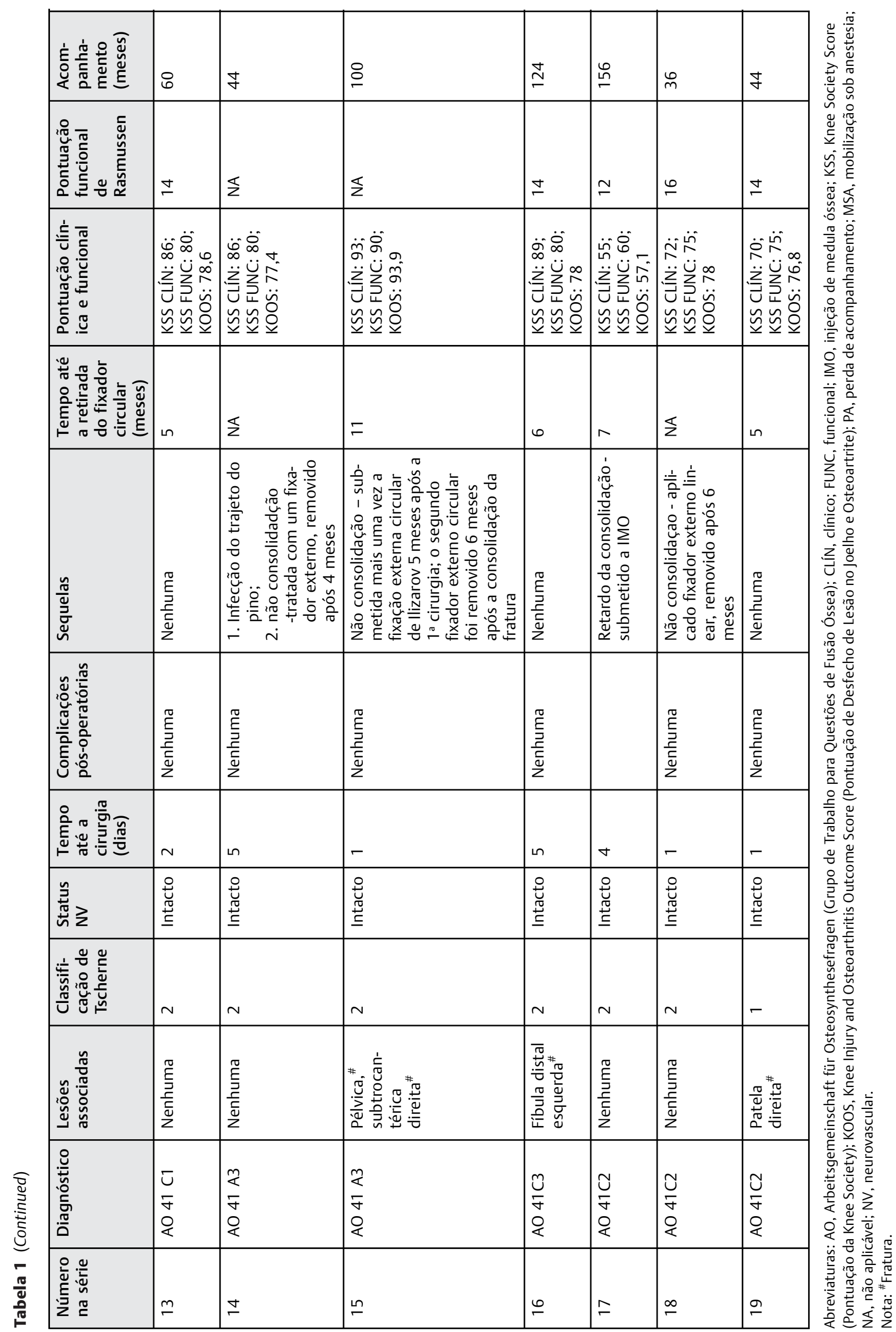




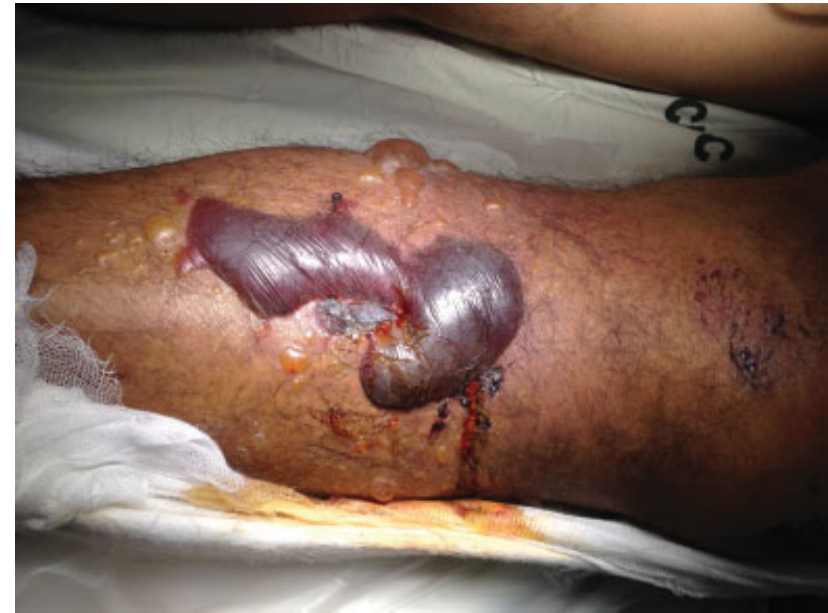

Fig. 1 Bolhas hemorrágicas.

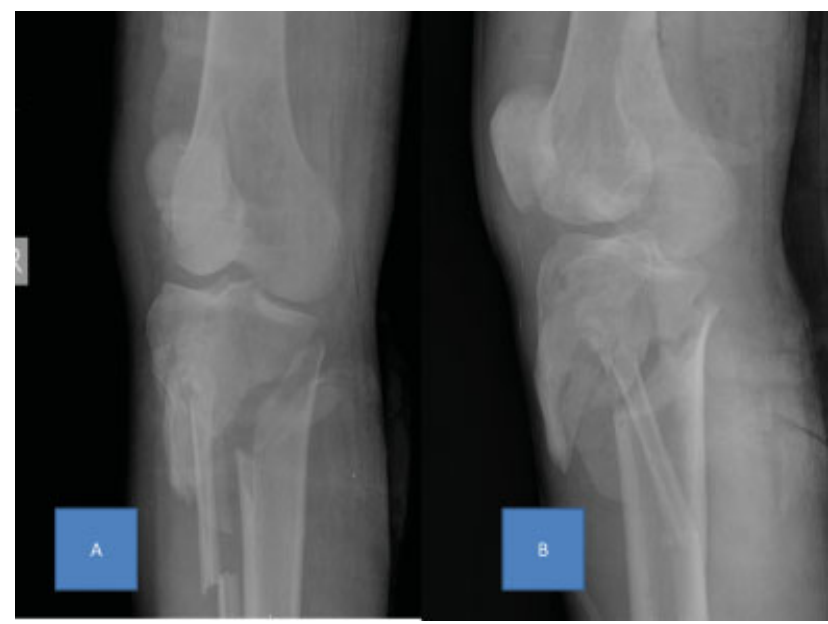

Fig. 2 A e 2b Radiografias pré-operatórias nas incidências anteroposterior e de perfil do Paciente 9.

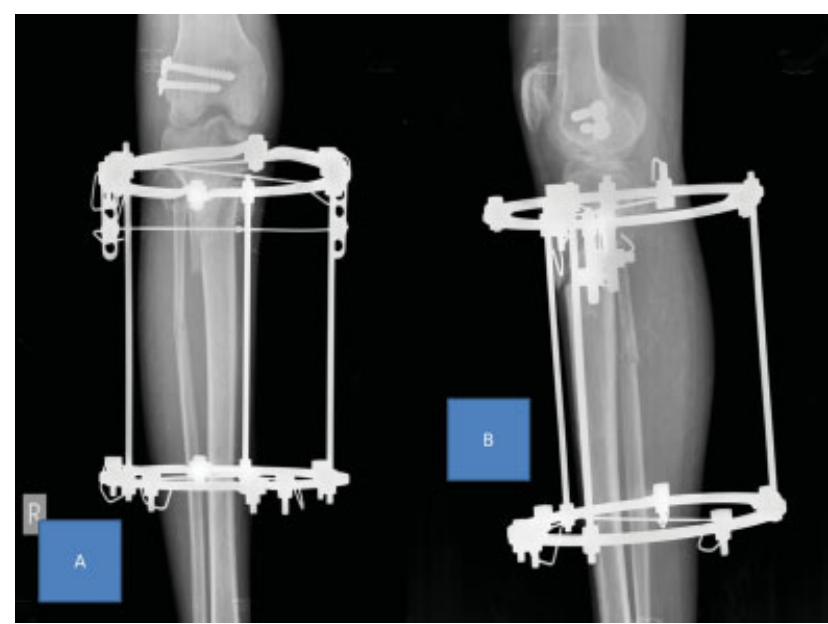

Fig. 3 A e 3B - Radiografias anteroposterior e de perfil do Paciente 9 no pós-operatório imediato com fixação de circular.

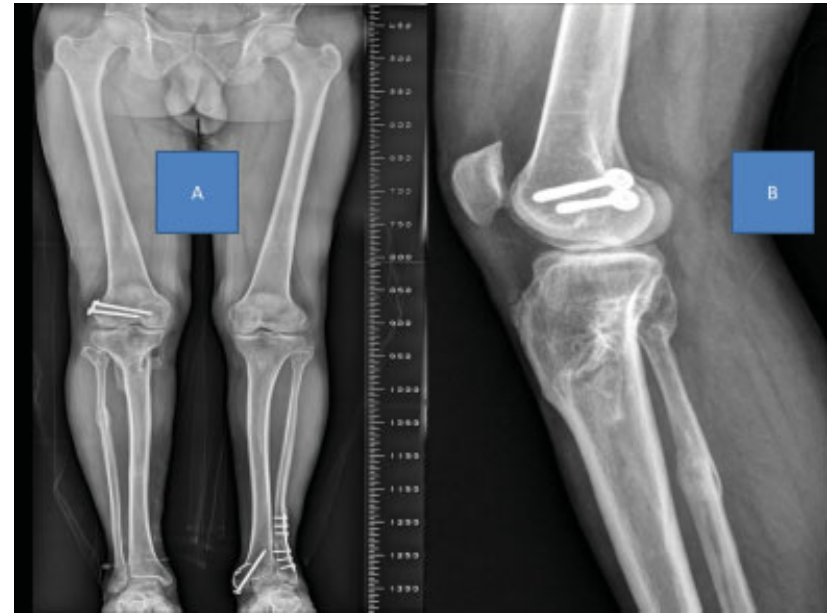

Fig. 4 A e 4B Radiografias anteroposterior e de perfil do Paciente 9 com 12 meses de acompanhamento.

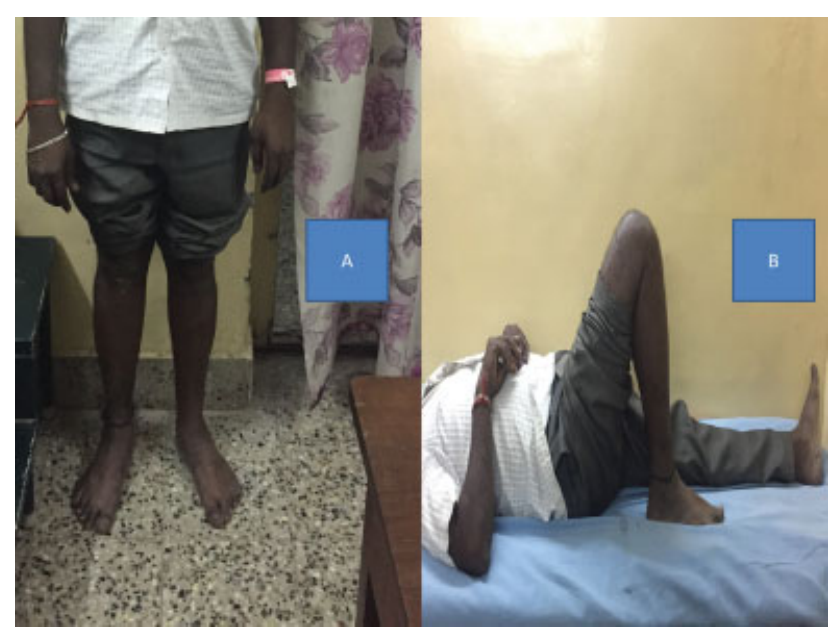

Fig. 5 A e 5B Paciente 9 com 12 meses de acompanhamento.

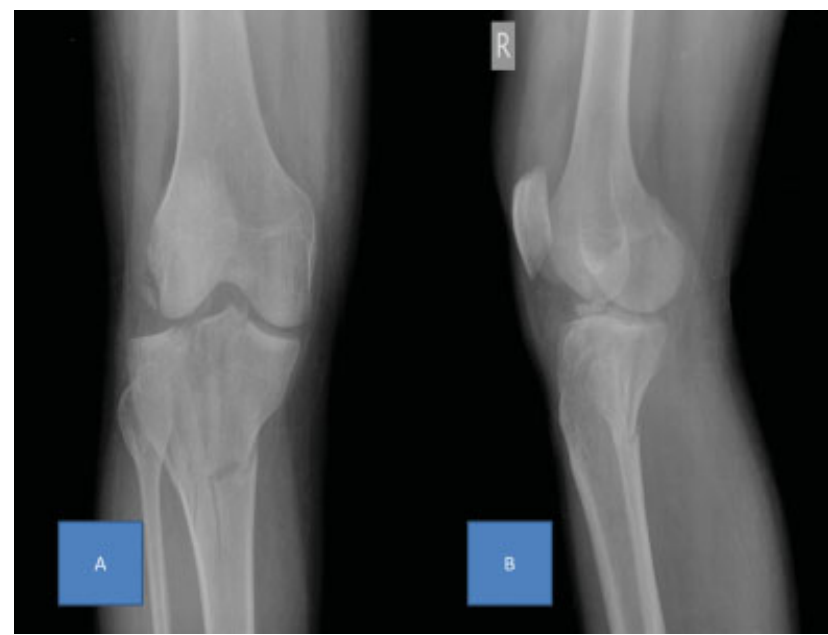

Fig. 6 A e 6B - Radiografias pré-operatórias anteroposterior e de perfil do paciente 13 . 


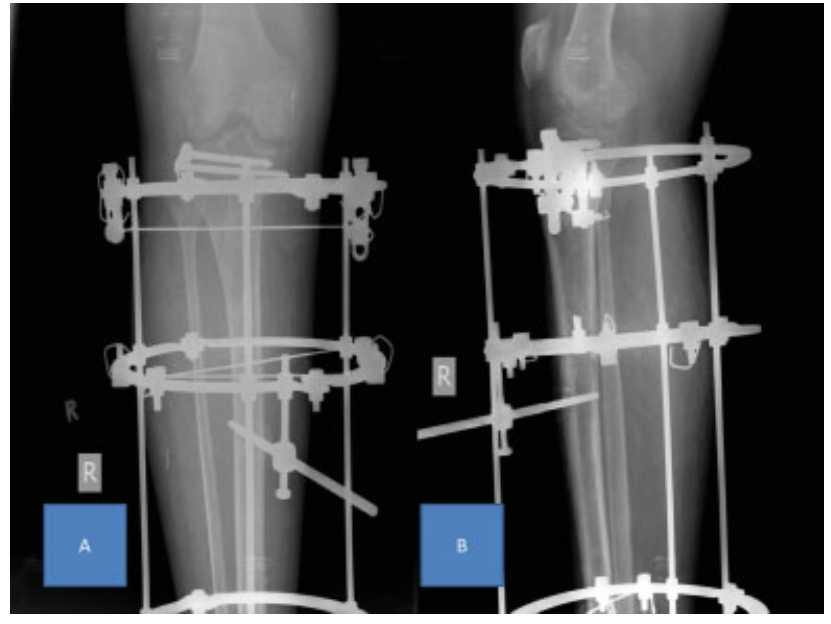

Fig. 7 A e 7B Radiografias anteroposterior e de perfil do Paciente 13 no pós-operatório imediato com fixação de circular.

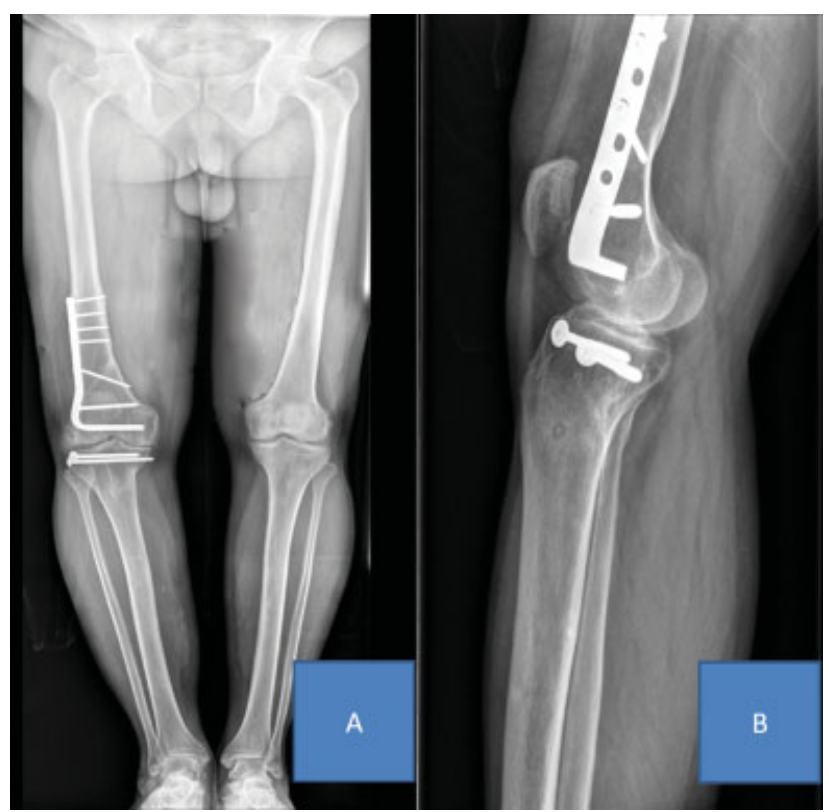

Fig. 8 A e 8B Radiografias anteroposterior e de perfil do Paciente 13 com 60 meses de acompanhamento.

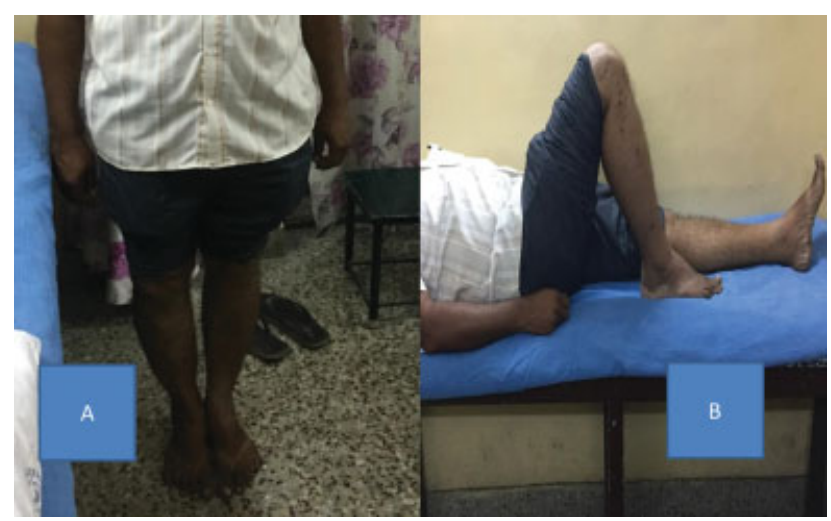

Fig. 9 A e 9B Paciente 13 com 60 meses de acompanhamento. de excelente a insuficiente. $O$ resultado médio na pontuação funcional de Rasmussen foi de $15 \pm 6,9$ em todos os pacientes com fratura intra-articular (—Figs. 6, 7, 8, 9).

\section{Complicações}

\section{Ósseas}

No total, 3 pacientes (16,7\%; Pacientes 14, 15 e 18) não se consolidaram após a fixação do aparelho; 2 desses pacientes foram submetidos à aplicação de um fixador externo linear, e 1 (Paciente 15) foi tratado novamente com um fixador externo circular de Ilizarov. Todos os três pacientes apresentaram consolidação no final do acompanhamento.

Retardo da consolidação foi diagnosticado em 2 pacientes (11,1\%; Pacientes 10 e 17), que foram tratados com injeção de medula óssea (IMO).

Osteomielite foi diagnosticada em 1 paciente (5,2\%; Paciente 17), que foi submetido a desbridamento 2 vezes após a consolidação da fratura.

\section{Partes Moles}

Um paciente desenvolveu rigidez no joelho (Paciente 9), e foi submetido a mobilização sob anestesia (MSA) no momento da remoção do anel.

No total, 2 pacientes (11,1\%; Pacientes 2 e 14) desenvolveram infecção no trajeto do pino, que foi tratada com curativos locais e antibióticos.

\section{Discussão}

O uso do fixador externo circular de Ilizarov é um método aceito de fixação para fraturas tibiais. Nas lesões tibiais proximais de alta energia, apresenta menor índice de complicações, mas resultados funcionais semelhantes quando comparados à fixação interna. ${ }^{14,15} \mathrm{~A}$ incidência da SCA nas fraturas da tíbia é de aproximadamente $4 \%$ a $11 \%{ }^{16,17}$ Discernir os sinais da SCI dos da SCA é a chave para um bom resultado clínico e funcional no tratamento dessas lesões. Os critérios clínicos estabelecidos por Sood et al. ${ }^{4}$ ajudam a diferenciar a SCI da SCA, pois a fasciotomia deve ser realizada com urgência na SCA.

Em nossa série de 19 pacientes, o diagnóstico de SCI foi feito logo no momento do comparecimento ao setor de emergência, sendo confirmado por 3 médicos experientes. Um dos métodos utilizados para identificar a gravidade do dano às partes moles é pela presença de bolhas hemorrágicas na tíbia proximal, que indica o desluvamento interno das partes moles. Então, aplicou-se imediatamente nesses pacientes medidas antiedema, e a fixação foi realizada com o aparelho de Ilizarov. A fixação interna não é recomendada nesses pacientes, pois o envelope de partes moles cicatriza em graus variados, levando à ruptura da pele quando é realizada a fixação interna.

Nenhum dos pacientes do grupo desenvolveu complicações em partes moles ou neurovasculares no pós-operatório imediato. A taxa de pseudoartrose no grupo foi de 16,7\% (3/18): 2 pacientes com fraturas extra-articulares e $1 \mathrm{com}$ fratura intra-articular com extensão diafisária. Esse valor foi baixo quando comparado com os da literatura 
disponível sobre os resultados das fraturas tibiais associadas à fasciotomia nas fraturas intra-articulares e diafisárias $(20 \% \text { a } 45 \%)^{2}$

Atualmente, existe uma preocupação com infecção após a fasciotomia e a redução aberta e fixação interna. A literatura recente ${ }^{5,15,18}$ mostra um aumento da incidência de infecção no sítio cirúrgico após a fixação interna em fratura do planalto tibial com síndrome compartimental. A cada dia de retardo no fechamento da ferida por fasciotomia, o risco de infecção aumenta em $7 \%{ }^{18}$ Sharma et al. $^{5}$ descreveram o tratamento de 15 pacientes, com fratura simples articular ou metafisária da tíbia proximal com $\mathrm{SCI}$, que foram tratados com fasciotomia e fixação interna em uma única etapa, dos quais 1 caso apresentou infecção superficial, e 2 casos apresentaram retardo na consolidação. Em nossa série de pacientes, nenhum desenvolveu infecção ou qualquer complicação nas partes moles no pós-operatório imediato. Todos os casos de SCI foram tratados de acordo com nosso protocolo de confirmação do diagnóstico por três médicos, que registraram de forma contínua e meticulosa todos os achados clínicos positivos e negativos, sendo utilizado o manitol a $20 \%$ para a redução das pressões compartimentais por 72 horas, além do do aparelho de Ilizarov para a fixação da fratura.

Ertürk et al. ${ }^{19}$ demonstraram uma diferença estatística significativa na pressão do compartimento quando a fixação interna foi comparada com a fixação do aparelho de Ilizarov em membros de coelhos, com uma redução da pressão do compartimento no grupo de fixação de Ilizarov. Eles atribuíram a queda de pressão no grupo de fixação externa circular à drenagem serosa por meio do trajeto dos pinos, que atuam como um conduto para a drenagem dos vários compartimentos. A redução do edema dos membros após a aplicação do aparelho de Ilizarov também é atribuída à estabilidade proporcionada pelo efeito da cama elástica. ${ }^{20}$ Neste estudo, utilizamos o fixador externo circular por acreditar que os fios e a armação geram um estado de estabilidade dinâmica no membro, evitando um aumento da pressão do compartimento, apesar da alteração no comprimento do osso e das partes moles.

Ulmer $^{21}$ mostrou que os sinais clínicos associados à SCA têm um alto valor preditivo negativo, o que indica que eles são os melhores a se usar para excluir a SCA. Usamos os critérios de Sood et al. ${ }^{4}$ para excluir a SCA e diagnosticar a SCI em todos os nossos pacientes. McQueen et al. ${ }^{22}$ reiteraram o papel da fasciotomia quando a pressão diferencial $(\Delta \mathrm{p})$ permanece $\leq 30 \mathrm{mmHg}$ por 2 horas seguidas. No entanto, a pressão compartimental e a medida da pressão diferencial no diagnóstico da SCI ou da SCA apresentam alta variabilidade interobservador, com variabilidade entre os métodos de medição, altas taxas de falso-positivo, e não estão disponíveis e e tampouco são utilizadas universalmente. ${ }^{23,24}$ Apesar dos avanços nos métodos não invasivos, como a dosagem dos níveis de creatina quinase $(\mathrm{CK})$ e a medição da elastância dos tecidos, com o intuito de aumentar a precisão do diagnóstico precoce da síndrome compartimental, ainda permanece uma falta de clareza em relação ao diagnóstico clínico da $\mathrm{SCI}^{21,25-27}$ Estudos $^{28}$ reiteraram a importância primária da avaliação clínica no tratamento da
$\mathrm{SCl}$. Acreditamos que o exame clínico rigoroso e repetitivo continua a ser a ferramenta mais importante no diagnóstico da SCI/SCA, diante da ausência de um manômetro em hospitais com recursos limitados ou com uso irregular. Atualmente, é prática comum combinar os pacientes de SCI com SCA, realizando a fasciotomia em todos, acumulando, assim, as complicações associadas ao tratamento cirúrgico das fraturas proximais tibiais de alta energia com as sequelas da fasciotomia. ${ }^{2}$

As outras opções para o tratamento dessas lesões são a aplicação de um fixador externo ou placa de gesso de Paris, até que a pele esteja suficientemente estável para que se realize a fixação interna, o que pode levar de 2 a 3 semanas. 0 fixador externo é considerado um fator de risco independente para infecção, mesmo em pacientes sem síndrome compartimental. ${ }^{29}$ A fixação interna escalonada não é possível em fraturas expostas e na ausência de pele intacta, devido à formação de bolhas de cicatrização descobertas. Acreditamos que a fixação do aparelho de Ilizarov tem um papel definitivo no tratamento dessas lesões causadas por SCI.

As vantagens deste estudo são que ele seguiu os critérios estabelecidos para o diagnóstico de SCI, utilizando um implante universalmente aceito no tratamento das fraturas da tíbia, que apresenta resultados clínicos, funcionais e radiológicos claros. Os destaques deste estudo são que desestimulamos enfaticamente o uso da fasciotomia como uma ferramenta no tratamento dos pacientes com SCI. Defendemos a documentação dos achados, as medidas clínicas, a fim de reduzir as pressões compartimentais e o fixador externo circular no tratamento dessas fraturas. As desvantagens deste estudo são o pequeno tamanho da amostra, a falta de um grupo controle, e o viés de recrutamento dos pacientes elegíveis. Esperamos que nossa experiência ajude a aumentar a consciência em relação ao tratamento da SCI, evitando as fasciotomias desnecessárias.

\section{Conclusão}

Os cirurgiões devem ser capazes de identificar a síndrome compartimental iminente e a estabelecida, pois a primeira pode ser tratada sem fasciotomia. A fixação com o aparelho de Ilizarov em fraturas de tíbia com ICS resulta em melhores resultados, com taxas de infecção e complicações de partes moles menores do que as resultantes após fasciotomia.

\section{Suporte Financeiro}

Não houve suporte financeiro de fontes públicas, comerciais, ou sem fins lucrativos.

Conflito de interesses

Os autores declaram não haver conflito de interesses.

\section{Referências}

1 McQueen MM, Christie J, Court-Brown CM. Acute compartment syndrome in tibial diaphyseal fractures. J Bone Joint Surg Br 1996; 78(01):95-98

2 Blair JA, Stoops TK, Doarn MC, et al. Infection and nonunion after fasciotomy for compartment syndrome associated with tibia 
fractures: a matched cohort comparison. J Orthop Trauma 2016; 30(07):392-396

3 Azar FM. Traumatic disorders. In: Canale ST, Beaty JH, editors, Campbell's Operative Orthopaedics. 12th ed. Philadelphia, PA, USA: Elsevier; 2013

4 Sood LK, Kanojia R, Vijay K, Mehra A, Sud A, Guglani A. Compartment syndrome - Early diagnosis and treatment. Indian J Orthop 2001;35(03):177-179

5 Sharma N, Singh V, Agrawal A, Bhargava R. Proximal tibial fractures with impending compartment syndrome managed by fasciotomy and internal fixation: A retrospective analysis of 15 cases. Indian J Orthop 2015;49(05):502-509

6 Ruedi T, Buckley R, Moran C, editors, AO principles of fracture management. New York: Thieme; 2007

7 Tiwari A, Haq AI, Myint F, Hamilton G. Acute compartment syndromes. Br J Surg 2002;89(04):397-412

8 Daniels M, Reichman J, Brezis M. Mannitol treatment for acute compartment syndrome. Nephron 1998;79(04):492-493

9 Lachiewicz PF, Funcik T. Factors influencing the results of open reduction and internal fixation of tibial plateau fractures. Clin Orthop Relat Res 1990;(259):210-215

10 Insall JN, Dorr LD, Scott RD, Scott WN. Rationale of the Knee Society clinical rating system. Clin Orthop Relat Res 1989;(248):13-14

11 Roos EM, Roos HP, Lohmander LS, Ekdahl C, Beynnon BD. Knee Injury and Osteoarthritis Outcome Score (KOOS)-development of a self-administered outcome measure. J Orthop Sports Phys Ther 1998;28(02):88-96

12 Rasmussen PS. Tibial condylar fractures. Impairment of knee joint stability as an indication for surgical treatment. J Bone Joint Surg Am 1973;55(07):1331-1350

13 Checketts RG, MacEachem AG, Otterbum M. Pin track infection and the principles of pin site care. In: De Bastiani G Apley AG, Goldberg AA, editors, Orthofix external fixation in trauma and orthopaedics. London: Springer; 2000:97-103

14 Canadian Orthopaedic Trauma Society. Open reduction and internal fixation compared with circular fixator application for bicondylar tibial plateau fractures. Results of a multicenter, prospective, randomized clinical trial. J Bone Joint Surg Am 2006;88(12):2613-2623

15 Metcalfe D, Hickson CJ, McKee L, Griffin XL. External versus internal fixation for bicondylar tibial plateau fractures: systematic review and meta-analysis. J Orthop Traumatol 2015;16(04):275-285

16 McQueen MM, Gaston P, Court-Brown CM. Acute compartment syndrome. Who is at risk? J Bone Joint Surg Br 2000;82(02):200-203
17 Shadgan B, Pereira G, Menon M, Jafari S, Darlene Reid W, O’Brien PJ. Risk factors for acute compartment syndrome of the leg associated with tibial diaphyseal fractures in adults. J Orthop Traumatol 2015;16(03):185-192

18 Dubina AG, Paryavi E, Manson TT, Allmon C, O'Toole RV. Surgical site infection in tibial plateau fractures with ipsilateral compartment syndrome. Injury 2017;48(02):495-500

19 Ertürk C, Altay MA, Altay N, et al. The effect of 2 different surgical methods on intracompartmental pressure value in tibial shaft fracture: An experimental study in a rabbit model. Ulus Travma Acil Cerrahi Derg 2017;23(02):85-90

20 Rudloff MI. Fractures of the Lower Extremity. In: Canale ST, Beaty JH, editors. Campbell's operative orthopaedics. Philadelphia: Elsevier Mosby; 2013:2617-2724

21 Ulmer T. The clinical diagnosis of compartment syndrome of the lower leg: are clinical findings predictive of the disorder? J Orthop Trauma 2002;16(08):572-577

22 McQueen MM, Duckworth AD, Aitken SA, Court-Brown CM. The estimated sensitivity and specificity of compartment pressure monitoring for acute compartment syndrome. J Bone Joint Surg Am 2013;95(08):673-677

23 Whitney A, O’Toole RV, Hui E, et al. Do one-time intracompartmental pressure measurements have a high false-positive rate in diagnosing compartment syndrome? J Trauma Acute Care Surg 2014;76(02):479-483

24 Collinge C, Kuper M. Comparison of three methods for measuring intracompartmental pressure in injured limbs of trauma patients. J Orthop Trauma 2010;24(06):364-368

25 Lollo L, Grabinsky A. Clinical and functional outcomes of acute lower extremity compartment syndrome at a Major Trauma Hospital. Int J Crit Illn Inj Sci 2016;6(03):133-142

26 Sellei RM, Hingmann SJ, Kobbe P, et al. Compartment elasticity measured by pressure-related ultrasound to determine patients "at risk" for compartment syndrome: an experimental in vitro study. Patient Saf Surg 2015;09(1):1-6

27 Schmidt AH. Continuous compartment pressure monitoring-better than clinical assessment? J Bone Joint Surg Am 2013;95(08): e52(1-2)

28 Bussell HR, Aufdenblatten CA, Subotic U, et al. Compartment pressures in children with normal and fractured lower extremities. Eur J Trauma Emerg Surg 2019;45(03):493-497

29 Parkkinen M, Madanat R, Lindahl J, Mäkinen TJ. Risk Factors for Deep Infection Following Plate Fixation of Proximal Tibial Fractures. J Bone Joint Surg Am 2016;98(15):1292-1297 